\title{
Geometry, electrostatic measure and orthogonal polynomials on Julia sets for polynomials
}

\author{
M. F. BARNSLEY, J. S. GERONIMO AND A. N. HARRINGTON
} School of Mathematics, Georgia Institute of Technology, Atlanta, GA 30332, USA

(Received 24 November 1982 and revised 6 June 1983)

Abstract. The Julia set $\mathrm{B}$ for an N'th degree polynomial $T$ and its equilibrium electrostatic measure $\mu$ are considered. The unique balanced measure on $B$ is shown to be $\mu$. Integral properties of $\mu$ and of the monic polynomials orthogonal with respect to $\mu, P_{n}, n=0,1,2, \ldots$, are derived. Formulae relating orthogonal polynomials of the second kind of different degrees are displayed. The measure $\mu$ is recovered both in the limit from the zeros and from the poles of the $\left[N^{n}-1 / N^{n}\right]$ Padé approximant to the moment generating function to $\mu$. For infinitely many polynomials of each degree $N$ the zeros and poles all lie on an increasing sequence of trees of analytic arcs contained in $B$. The properties of these Padé approximant sequences support conjectures of George Baker which have not previously been tested on measures supported on sets nearly as complicated as Julia sets spread out in the complex plane.

\section{Introduction}

This paper centres on the Julia set $B$ for a complex polynomial of degree $N \geq 2$,

$$
T(z)=z^{N}+k_{1} z^{N-1}+\cdots+k_{N}
$$

(see [12], [15], [18], [19]). Let

$$
T^{0}(z)=z, \quad T^{n}(z)=T \circ T^{n-1}(z),
$$

$n=1,2, \ldots B$ is the set of points where $\left\{T^{n}(z)\right\}_{n=0}^{\infty}$ is not normal. $B$ is compact and perfect. $T^{-1}(B)=B$. $B$ may be connected, disconnected, or totally disconnected. Except in special cases no lines are tangent to $B$.

Associated with $B$ are: the electrostatic measure $\mu$ on $B$ with $\mu(B)=1,[8],[12]$; the monic $n$ 'th degree polynomials $P_{n}(z), n=0,1,2, \ldots$, which are orthogonal under the Hermitian inner product

$$
\langle f, g\rangle=\int_{B} f(z) \overline{g(z)} d \mu(z) ;
$$

the polynomials of the second kind,

$$
P_{n}^{(1)}(z)=\int_{B} \frac{P_{n+1}(z)-P_{n+1}(w)}{z-w} d \mu(w) ;
$$


and the Padé approximants (see [1]) for the moment generating function

$$
G(z)=\int_{B} \frac{d \mu(w)}{z-w} .
$$

We will derive properties of the above functions. For a subclass of polynomials $T$ with real coefficients, we relate subsequences of the orthogonal polynomials and Padé approximants to the geometry of the Julia set $B$ and associated trees of analytic arcs. In particular, we give examples of Padé approximants supporting conjectures of George Baker and others ([1]). We derive properties of $\mu$ in $\S 1$ and of polynomials $P_{n^{N-1}}^{(1)}$ in $\S 2$. In $\S \S 3$ and 4, we generalize [4], [6] relating the geometry of some Julia sets to orthogonal polynomials and Padé approximants. In contrast to $\$ \S 1-4$ dealing with $N$ 'th degree polynomials, we include in an appendix a result we have only proved so far for $N=2$. When the Julia set $B$ is real, we calculate upper and lower bounds on norms of $\boldsymbol{P}_{n}$.

The study of orthogonal polynomials on Julia sets is of interest for a variety of reasons. Overall, this area of research involves and relates a wide range of mathematical topics including analytic function theory, ergodic theory, orthogonal polynomials and spectra of operators, [7], moment theory, and iterated maps and dynamical systems. It is also related to models in mathematical physics including models for chaotic phenomena and turbulence in deterministic systems [13], model Schrödinger equations for crystal structures involving almost-periodic potentials [9], and Ising model lattice gases [3].

Some related results concerning the calculation of moments and Padé approximants are described in [10] and [11], using the Böttcher equation as the basic mechanism. Such an approach does not apply to arbitrary rational functions, whereas the one given in the present paper does, [2].

\section{Properties of the measure $\mu$}

The first part of this section is a uniqueness theorem for the balanced measures introduced in [5]. Next we derive a consequence of $\mu$ being balanced which we call quick mixing. We use quick mixing to obtain general integral formulae. We shall often use a shorthand notation for integrals involving $\mu$ :

$$
I(f)=\int_{B} f(z) d \mu(z) .
$$

For example, $\langle f, g\rangle=I(f \bar{g})$.

The following definition and theorem 1 give an alternative description of the electrostatic equilibrium measure $\mu$ discussed by Brolin [12].

A probability measure $\sigma$ on $B$ is a balanced measure for $T$ if

$$
\sigma\left(T_{j}^{-1}(S)\right)=\frac{1}{N} \sigma(S)
$$

for each Borel subset $S$ of $\mathbb{C}$ and each branch $T_{j}^{-1}, j=1,2, \ldots, N$ of $T$.

THEOREM 1. There is a unique balanced measure for $T$ on $B$. It is the electrostatic equilibrium measure $\mu$. 
Proof. Let $\sigma$ be a balanced measure for $T$ on $B$. In order to apply a result of Brolin, we cover $B$ with a grid of arbitrarily small square regions $Q$ such that $\mu(\partial Q)=0$ for each square region $Q$. It is sufficient to show $\mu(Q)=\sigma(Q)$ for an arbitrary grid square. Let $f$ be the characteristic function for such a $Q$. Since $\sigma$ is balanced we have, [5],

$$
\sigma(Q)=\int_{B} f(z) d \sigma(z)=\int_{B} \frac{1}{N} \sum_{j=1}^{N} f\left(T_{j}^{-1}(z)\right) d \sigma(z) .
$$

Iterating we obtain

$$
\sigma(Q)=\int_{B} \frac{1}{N^{m}} \sum_{j=1}^{N^{m}} f\left(T_{j}^{-m}(z)\right) d \sigma(z),
$$

where $T_{j}^{-m}, j=1,2, \ldots, N^{m}$ are the branches of the inverse of $T^{m}$. Brolin $([12$, proof of theorem 17.1]) showed that

$$
\frac{1}{N^{m}} \sum_{j=1}^{N^{m}} f\left(T_{j}^{-m}(z)\right) \rightarrow \int_{B} f(w) d \mu(w)
$$

uniformly for $z \in B$ as $m \rightarrow \infty$. Hence we can let $m \rightarrow \infty$ in (1) to finish the proof:

$$
\begin{aligned}
\sigma(Q) & =\int_{B}\left(\int_{B} f(w) d \mu(w)\right) d \sigma(z) \\
& =\int_{B} f(w) d \mu(w) \cdot 1=\mu(Q) .
\end{aligned}
$$

Demko [14] has proved recently the existence of balanced measures in a very general setting.

Brolin [12] proved that $T$ is mixing on $B$ with respect to $\mu$. We show now that $\mu$ and $T$ satisfy a related property which we call quick mixing, indicated by the following theorem.

THEOREM 2. If $f \in L^{1}(B, \mu)$ and $Q$ is a polynomial of degree less than $N^{n}$, then

$$
\int_{B} f\left(T^{n}(z)\right) Q(z) d \mu(z)=\int_{B} f(z) d \mu(z) \int_{B} Q(z) d \mu(z) .
$$

In shorthand notation,

$$
I\left(\left(f \circ T^{n}\right)(Q)\right)=I(f) I(Q) .
$$

Proof. Using the balanced property of $\mu$ we showed [5] that

$$
\int_{B} z^{j} f(T(z)) d \mu(z)=\frac{S_{j}}{N} \int_{B} f(z) d \mu(z),
$$

where the symmetric function $S_{j}=-j k_{j}-\sum_{l=1}^{j-1} k_{l} S_{l}, j=1,2, \ldots, N-1$, with $k_{j}$ the coefficient of $z^{N-j}$ in $T$. We can also allow $j=0$ in (3) setting $S_{0}=N$. Here as elsewhere, we use the notation $T^{n}$ for the $n$ 'th iterate of $T$; however, when we wish to denote a power of a function, we shall always use a parenthesis - for example $\left(T^{2}\right)^{3}$ means the second iterate of $T$ raised to the third power.

The set $\mathscr{P}$ of polynomials of the form

$$
\prod_{j=0}^{n-1}\left(T^{j}(z)\right)^{m_{i}}, \quad m_{j} \in\{0,1, \ldots, N-1\}
$$


contains a polynomial of each degree $0,1, \ldots, N^{n}-1$ so $Q$ lies in the span of $\mathscr{P}$. It is sufficient to assume $Q \in \mathscr{P}$. We may use (3) repeatedly to complete the proof:

$$
\begin{aligned}
I\left((Q)\left(f \circ T^{n}\right)\right) & =I\left(\left(\prod_{i=0}^{n-1}\left(T^{j}\right)^{m_{j}}\right)\left(f \circ T^{n}\right)\right) \\
& =\left(\prod_{j=0}^{n-1} \frac{S_{m_{j}}}{N}\right) I(1) I(f) \\
& =I\left(\prod_{j=0}^{n-1}\left(T^{j}\right)^{m_{i}}\right) I(f)=I(Q) I(f) .
\end{aligned}
$$

Theorem 2 simplifies a variety of integral calculations as the following theorem illustrates.

THEOREM 3. If $f \in L^{1}(B, \mu)$ then

$$
\int_{B} P_{l}(z) f\left(T^{n}(z)\right) d \mu(z)=0
$$

where $l<N^{n+1}, N^{n}$ does not divide $l$, and $P_{l}$ is an orthogonal polynomial defined in the introduction.

Proof. We can expand

$$
P_{l}=\sum_{m=0}^{\left[l / N^{n}\right]} P_{m}\left(T^{n}\right) \pi_{m},
$$

where $\pi_{m}$ is some polynomial of degree $<N^{n}$. Using quick mixing, we find

$$
I\left(\left(P_{l}\right)\left(f \circ T^{n}\right)\right)=\sum_{m=0}^{\left[l / N^{n}\right]} I\left(P_{m} f\right) I\left(\pi_{m}\right) .
$$

We can let $f=\vec{P}_{j}, j=0,1,2, \ldots,\left[l / N^{n}\right]$ in (4) so $([5]) f \circ T^{n}=\overline{P_{j N^{n}}}$. We can use the orthogonality of $P_{j N^{n}}$ and $P_{l}$ to obtain

$$
0=\left\langle P_{l}, P_{j} \circ T^{n}\right\rangle=\left\langle P_{j}, P_{j}\right\rangle I\left(\pi_{j}\right),
$$

so $I\left(\pi_{j}\right)=0, j=0,1,2, \ldots,\left[l / N^{n}\right]$. On substitution into (4), the theorem follows.

Theorem 3 allows an easy proof of theorem 4 .

THEOREM 4. Let $m \neq n$. Then

$$
\int_{B} P_{N^{n}}(z) P_{N^{m}}(z) d \mu(z)=0
$$

and also

$$
\begin{aligned}
0 & =\left\langle\operatorname{Re}\left(P_{N^{n}}\right), \operatorname{Re}\left(P_{N^{m}}\right)\right\rangle=\left\langle\operatorname{Re}\left(P_{N^{n}}\right), \operatorname{Im}\left(P_{N^{m}}\right)\right\rangle \\
& =\left\langle\operatorname{Im}\left(P_{N^{n}}\right), \operatorname{Im}\left(P_{N^{m}}\right)\right\rangle .
\end{aligned}
$$

If $T$ has real coefficients, then

$$
\left\langle\operatorname{Re}\left(P_{N^{n}}\right), \operatorname{Im}\left(P_{N^{n}}\right)\right\rangle=0 .
$$

Proof. Take $n>m$ and (5) is a special case of theorem 3 with $f(z)=\bar{z}$. The next line of orthogonality relations follows using the facts that both the inner products 
of $P_{N^{n}}$ with $P_{N^{m}}$ and of $P_{N^{n}}$ with $\overline{P_{N^{m}}}$ are 0 . Finally, if the coefficients of $T$ are real then $B, \mu$, and $P_{j}$ are symmetric across the real axis and

$$
0=\operatorname{Im}\left(I\left(\frac{1}{2} P_{j}^{2}\right)\right)=\left\langle\operatorname{Re}\left(P_{j}\right), \operatorname{Im}\left(P_{j}\right)\right\rangle .
$$

Remark. In the special case $T(z)=z^{N}, \mu$ is a uniform measure on the unit circle and all the integrals reduce to classical orthogonality relations for

$$
\operatorname{Re}\left(P_{N^{n}}\left(e^{i \theta}\right)\right)=\cos \left(N^{n} \theta\right) \text { and } \operatorname{Im}\left(P_{N^{m}}\left(e^{i \theta}\right)\right)=\sin \left(N^{m} \theta\right) .
$$

The theorem gives us a broad class of generalizations.

\section{Orthogonal polynomials of the second kind and Páde approximants}

We prove a recursion relation for $P_{m N-1}^{(1)}$ and $P_{m-1}^{(1)}$ and a corresponding relation for Padé approximants to $G(z)$ about $\infty$. The Padé approximant denoted by $[\mathrm{m} / \mathrm{n}]$ has numerator polynomial $P$ of degree at most $m$ and denominator polynomial $Q$ of degree at most $n$, where these satisfy

$$
P(z)-G(z) Q(z)=O\left(1 / z^{m+n+1}\right) .
$$

THEOREM 5.

$$
\begin{gathered}
P_{m N-1}^{(1)}(z)=\frac{T^{\prime}(z)}{N} P_{m-1}^{(1)}(T(z)) \\
P_{m N^{n}-1}^{(1)}(z)=\frac{1}{N^{n}} \frac{d}{d z}\left(T^{n}(z)\right) P_{m-1}^{(1)}\left(T^{n}(z)\right) \\
=\frac{1}{N^{n}}\left(\prod_{j=0}^{n-1} T^{\prime}\left(T^{j}(z)\right) P_{m-1}^{(1)}\left(T^{n}(z)\right)\right)
\end{gathered}
$$

Corollary. Padé approximants to $\int_{B} d \mu(w) /(z-w)$ satisfy

$$
[m N-1 / m N](z)=\frac{T^{\prime}(z)}{N}[m-1 / m](T(z))
$$

and

$$
\left[m N^{n}-1 / m N\right](z)=\frac{d}{d z^{n}}\left(\frac{T^{n}(z)}{N^{n}}\right)[m-1 / m]\left(T^{n}(z)\right) .
$$

Proof. We can rewrite

$$
\begin{aligned}
P_{m N-1}^{(1)}(z) & =\int_{B} \frac{P_{m N}(z)-P_{m N}(w)}{z-w} d \mu(w) \\
& =\int_{B} \frac{P_{m}(T(z))-P_{m}(T(w))}{z-w} d \mu(w),
\end{aligned}
$$

since $P_{n}(T(z))=P_{n N}(z)$ ([5]). Using the balanced property of $\mu$ yields

$$
P_{m N-1}^{(1)}(z)=\frac{1}{N} \int_{B} P_{m}(T(z))-P_{m}(w) \sum_{j=1}^{N} \frac{1}{z-T_{j}^{-1}(w)} d \mu(w) .
$$

But

$$
\sum_{j=1}^{N} \frac{1}{z-T_{j}^{-1}(w)}=\frac{T^{\prime}(z)}{T(z)-w},
$$

and (7) follows directly, and (8), by induction. 
When the Julia set lies on the real line, the corollary is a consequence of the theorem and the fact that the $[n-1 / n]$ Padé approximant can be written as, $[1]$,

$$
[m-1 / m](z)=P_{m-1}^{(1)}(z) / P_{m}(z) \text {. }
$$

With some further analysis the formalism used when the Julia set is real can be adapted for when $B$ is in the complex plane. The coefficients in Padé approximants to $G$ are obtained from linear equations involving the moments $\int w^{n} d \mu(z), n=$ $0,1,2, \ldots$ The reduction formula (3) allows us to calculate each moment, and they all are polynomials in the coefficients of $T$. Consequently, the coefficients in the Padé approximants are all rational functions of the coefficients of $T$. For each degree $N$ there is an open set in $\mathbb{R}^{N}$ of coefficient vectors $\left(k_{1}, k_{2}, \ldots, k_{N}\right)$ such that the Julia set for $T$ is a generalized Cantor set in $\mathbb{R}$, see theorem 6 . On this open set in real parameter space, the corollary holds, saying that the rational functions on both sides of (9) and (10) are equal. Hence, they agree for any complex values of $\left(k_{1}, k_{2}, \ldots, k_{N}\right)$.

Remark. Note that non-trivial situations where infinite subsequences of Padé approximants can be calculated and analyzed in detail are rare. We now have concrete examples of Padé approximants associated with totally singular measures supported not only on subsets of lines and circles, but also on complicated sets in the plane.

\section{Geometry of $B$ when $T$ has real coefficients and real critical points}

In this section we lead up to a discussion of trees of analytic arcs associated with some connected Julia sets. The trees are contained in $B$ for some $T$. First we collect some basic possibilities in:

THEOREM 6. Let $B$ denote the Julia set for

$$
T(z)=z^{N}+k_{1} z^{N-1}+\cdots+k_{N}
$$

where $k_{1}, k_{2}, \ldots, k_{N}$ are real, and $T$ has a largest real fixed point $a=T(a)$. If $N$ is odd let $b$ be the smallest real fixed point. If $N$ is even let $b$ be the smallest real pre-image of $a$. Then $a$ and $b$ are the largest and smallest real points on $B$.

Suppose that all the roots $z_{j}$ of $T^{\prime}$ are real. We distinguish three cases:

(1) $T\left(z_{j}\right) \in[b, a]$ for $j=1,2, \ldots, N-1$. Then $B$ is connected. Let $I_{0}=[b, a]$, $I_{k}=T^{-1}\left(I_{k-1}\right), k=1,2, \ldots$,

$$
K=\bigcup_{k=0}^{\infty} I_{k} .
$$

Then $I_{k}$ is a finite tree of analytic arcs and $B \subset K \subset B \cup\{$ bounded components of the complement of $B$ \}.

(2) $T\left(z_{j}\right) \notin[b, a]$ for some $j$. Then $B$ is not connected.

(3) $T\left(z_{j}\right) \notin[b, a]$ for $j=1,2, \ldots, N-1$. Then $B \subset[b, a]$ and is totally disconnected.

Proof. Clearly if $x>a, \lim _{n \rightarrow \infty} T^{n}(x)=\infty$. Since $f^{\prime}(a) \geq 1$, the fixed point $a$ lies on $B$, [12]. A similar argument holds when $x<b$. 
(1) Suppose $T\left(z_{j}\right) \in[b, a], j=1,2, \ldots, N-1$. By this assumption all branch points of $T^{-1}$ lie on $[b, a]=I_{0}$, so $T^{-1}\left(I_{0}\right)=I_{1}$ will be a tree of analytic arcs. Because the endpoints of $I_{0}$ are fixed points in $I_{0}$ or the pre-image of a fixed point in $I_{0}$, $I_{1} \supset I_{0}$. Hence if we define inductively

$$
I_{j}=T^{-1}\left(I_{j-1}\right), \quad j=1,2, \ldots,
$$

we find that $I_{j}$ is a tree of analytic arcs containing $I_{j-1}$. Now let

$$
H_{0}(z)=\frac{a-b}{4}\left(z+\frac{1}{z}\right)+\frac{a+b}{2}
$$

which maps $D_{0}=\{z:|z|>1\}$ conformally onto the complement of $I_{0}$. Because of the location of the branch points of $T^{-1}$ on $I_{0} \subset I_{j}$ we may define inductively one-to-one analytic functions $H_{j}$ on $D_{0}, j=1,2, \ldots$, by

$$
T\left(H_{j}(z)\right)=H_{j-1}\left(z^{N}\right)
$$

where $H_{j}(z)=((a-b) / 4)^{N^{-j}} z+O(1)$ at $\infty$, and the image of $H_{j}$ is the complement of $I_{j}$

From the normalization at $\infty$ and the monotonicity of the images, $H_{k}$ converges to a one-to-one analytic function, call it $H$, on $D_{0}$. As discussed by Fatou [15], the boundary of $H\left(D_{0}\right)$ is $B$. From the monotonicity we obtain

$$
K=\bigcup_{j=0}^{\infty} I_{j} \subset B \cup\{\text { bounded components of the complement of } B\} \text {. }
$$

That $B \subset K$ follows since $K$ is closed and contains the pre-images of all orders of $a$ (see [12]).

In (2) and (3) some or all of the branch points of $T^{-1}$ are attracted to $\infty$ so the conclusions about disconnectedness follow from [12]. Finally we note that in case (3) $T^{-1}([b, a]) \subset[b, a]$ so $B \subset[b, a]$.

We now show that there are infinitely many cases when $B$ is the closure of the union of the trees $I_{j}$.

THEOREM 7. Let $\mathscr{T}$ be the set of all monic $N$ 'th degree polynomials $T$ with real coefficients such that if $T^{\prime}(z)=0$ then $z \in B \cap \mathbb{R}$. There are infinitely many polynomials in $\mathscr{T}$ whose coefficients are all arbitrarily close to those of the Chebychev polynomials of degree $N$ on $[-2,2]$. If $T \in \mathscr{T}$, then the complement of $B$ is connected, and $B=K$ where $K$ is described in theorem 6.

Remark. We see that $B$ has a very special structure when $T \in \mathscr{T}$, but this restriction on $T$ is satisfied in many cases of interest. Real iterated maps of an interval have received much study. The hypothesis of real coefficients for $T$ allows us to restrict attention to the real line, if we desire, and the condition on the critical points of $T$ ensures that $T$ maps the interval $[b, a]$ into itself. Grebogi, Ott, and Yorke [16] have studied the mixing properties of a quadratic $T \in \mathscr{T}$. Jakobson [17] has also considered the case $N=2$ and his work shows that if $T(z)=(z-\lambda)^{2}$ then $T$ is in $\mathscr{T}$ for a set of $\lambda$ values of positive measure. 
Proof of theorem 7. Let

$$
T(z)=T_{d}(z)=\frac{1}{d^{N}} C(d z)
$$

where $C$ is the $N$ 'th degree Chebychev polynomial on $[-2,2]$. We shall show that there are values of $d>1$ arbitrarily close to 1 such that $T_{d} \in \mathscr{T}$. Let $p_{0}$ be a fixed point of $T_{d}$ other than $\pm a$. For $d$ close enough to $1, p_{0}$ is repulsive and therefore in $B$. There is a branch $R$ of $T_{d}^{-1}$ with $a$ as a fixed point such that the points $p_{k}=R\left(p_{k-1}\right), k=1,2, \ldots$, converge to $a$ as $k \rightarrow \infty$. All the points $\pm p_{k}$ lie on $B$ and are continuous functions of $d$ and have magnitude $<a$ for $d \geq 1$. The magnitude of the local maxima and minima of $T_{d}$ are all equal and increase to $a$ continuously as $d \rightarrow 1^{+}$, so they pass through an infinite number of the points $p_{n}$ in the process. When the local maxima or minima equal $P_{n}, T_{d} \in \mathscr{T}$.

Next we show that for $T \in \mathscr{T}$ there can be no bounded component $D$ of the complement of $B$, so the complement of $B$ consists of the one component containing $\infty$. If $D$ exists, Sullivan [21] has shown that there exist $j, k>0$ such that

$$
T^{j+k}(D)=T^{j}(D) \text {. }
$$

$T^{j}(D)$ must then either (i) contain a branch point for $T$, or (ii) be a rotation domain. For $T \in \mathscr{T}$ all branch points lie in $B$, so (i) is impossible. Possibility (ii) is excluded because all iterates of the branch points lie on the real line.

Theorem 6 and the non-existence of a bounded domain $D$ allow us to draw the final conclusion in theorem 7 , that $K=B$.

A simple consequence of theorem 7 together with Mergelyan's Theorem [20], is that for $T$ in $\mathscr{T}$, the set of orthogonal polynomials $\left\{P_{n}\right\}$ is dense in the continuous functions on $B$. Hence, the orthogonal polynomials are also dense in $L^{2}(B, \mu)$.

\section{Padé approximants and orthogonal polynomials in relation to the geometry of $B$} We start with several properties of orthogonal polynomials $P_{N^{n}}$ and end with a description of the asymptotic distribution of zeros and poles for a sequence of Padé approximants to the moment generating function for $\mu$.

THEOREM 8. If $T$ is an even or odd polynomial, then $P_{N^{n}}$ attains its maximum modulus on $B$ on a set containing at least $N^{n}+1$ points, $n=0,1,2, \ldots$

Proof. Let $M_{n}$ be the set of points at which $P_{N^{n}}$ attains its maximum modulus, $n=0,1,2, \ldots$ If $T$ is even or odd, then $B$ is symmetric about 0 , and $P_{1}(z)=z$. $M_{1}$ will at least contain two opposite points, so the theorem is true for $P_{1}$.

Suppose the theorem is true for $P_{N^{n-1}}$ so $M_{n-1}$ has at least $N^{n-1}+1$ points.

$$
P_{N^{n}}(z)=P_{N^{n-1}}(T(z))
$$

so

$$
M_{n}=T^{-1}\left(M_{n-1}\right) \text {. }
$$

$M_{n}$ contains at least $N\left(N^{n-1}+1\right)$ points counting multiplicity and at least

$$
N\left(N^{n-1}+1\right)-(N-1)=N^{n}+1
$$

not counting multiplicity, so the proof by induction is complete. 
Remark. The conclusion of theorem 8 is necessary for $P_{N^{n}}$ to be a Chebychev polynomial, but not sufficient. It follows from [6] that when $T(z)=z^{2}-\lambda, P_{2^{N}}(z)$ is a Chebychev polynomial on $B$, (namely, the unique polynomial of degree $2^{N}$ and leading coefficient one, with minimum supremum norm over $B$ ). This proof can easily be generalized to the case where $T(z)=z^{N}-\lambda$, or even where $T$ is a composition of such functions with differing values of $N$ and $\lambda$.

Remark. If no multiple points occur in the first $n$ steps, we see that $M_{n}$ contains $2 N^{n}$ points or more. No multiple points will occur if $T^{j}(z)$ is not a point of $B$ with maximum modulus for any root $z$ of $T^{\prime}$ and for $j=1,2, \ldots n$. On the other extreme, our lower bound $N^{n}+1$ for points in $M_{n}$ is sharp if $T$ is the $N^{\prime}$ th degree Chebychev polynomial for the interval $[-2,2]$.

We note one further special property of $P_{N^{n}}$.

THEOREM 9. $P_{N^{n}}$ is a Faber polynomial for the function $F$ which solves the Böttcher functional equation at $\infty$ :

$$
(F(z))^{N}=F(T(z)) \quad \text { with } F(z)=z+O(1) \text { at } \infty .
$$

Proof. Immediate from theorem 4 of [5]:

$$
\left(P_{N^{n}}(z)\right)^{1 / N^{n}}=F(z)\left(1+O\left(z^{-2 N^{n}}\right)\right) .
$$

Remark. If $B$ is connected, $F$ is a conformal mapping between the regions containing $\infty$ bounded by $B$ and by the unit circle respectively. See [15] and [8] for more on the Böttcher equation.

We now collect results on Padé approximants and $B$.

THEOREM 10. Suppose $T(z)=z^{N}+k_{1} z^{N-1}+\cdots k_{N}$ has real coefficients, $T^{\prime}(z)$ has all real roots, and the Julia set $B$ for T is connected. Let $G(z)$ be the moment generating function for the equilibrium measure $\mu$ on $B$,

$$
G(z)=\int_{B} \frac{d \mu(w)}{z-w}
$$

The $\left[N^{n}-1 / N^{n}\right]$ Padé approximant to $G(z)$ about $\infty$ is

$$
\frac{1}{N^{n}} \frac{\prod_{j=0}^{n-1} T^{\prime}\left(T^{j}(z)\right)}{T^{n}(z)+k_{1} / N}, \quad n=1,2, \ldots
$$

With the notation of theorems 6 and 7 , the zeros of the above numerator are the points on $I_{n-1}$ where $I_{n}$ branches. The zeros of the denominator also lie on $I_{n}$. The residues of the quotient are proportional to the orders of the roots of the denominator. If $T(z) \neq z^{N}$, the normalized counting measures for the roots of the denominator converge weakly to $\mu$ as $n \rightarrow \infty$. Similarly for the zeros of the numerator. When $T \in \mathscr{T}$ the zeros and poles all lie on $B$.

Proof. The equation for the Padé approximants comes from theorem 5 and its corollary. The location of the roots and poles follows from the construction of the $I_{n}$ 's in the proof of theorem 6 . The form of the residues is that of any logarithmic 
derivative. The statements about the measure follow from Brolin's ([12]) constructions of $\mu$.

Remark. Although Baker [1] has hypotheses about pole control for infinite sequences of Padé approximants to integrals over general sets in the complex plane, there is a dearth of theorems and even examples where the integral is not over a subset of a line or a circle. Theorem 10 supplies detailed information about zeros and poles for a wide class of examples.

\section{Appendix}

We specialize $T$ in this appendix to be $T(z)=(z-\lambda)^{2}, \lambda>2$, and we obtain upper and lower bounds on norms of the orthogonal polynomials. Let

$$
\left\|P_{n}\right\|=\left\langle P_{n}, P_{n}\right\rangle^{\frac{1}{2}}, \quad\left\|P_{n}\right\|_{\infty, B}=\sup _{x \in B}\left|P_{n}(z)\right| .
$$

THEOREM. Let $T(z)=(z-\lambda)^{2}, \lambda>2$. Then

$$
\begin{aligned}
& \left\|P_{2^{n}-1}\right\|^{2} \geq \lambda(\lambda-1)^{n} \quad n=1,2, \ldots \text {; } \\
& \left\|P_{2^{n}+s}\right\| \leq\left\|P_{2^{n}+s}\right\|_{\infty, B} \leq c\left(\frac{1+\lambda}{c}\right)^{n}, \quad \begin{aligned}
n & =0,1,2, \ldots, \\
s & =0,1,2, \ldots, 2^{n},
\end{aligned}
\end{aligned}
$$

where $c=\frac{1}{2}+\sqrt{\lambda+\frac{1}{4}}=c^{2}-\lambda$.

Proof. We use results from [4]:

$B$ is real, its largest and smallest points are

$$
\begin{gathered}
\lambda-c \text { and } \lambda+c ; \lambda-c>0 . \\
\quad P_{2 m}(z)=P_{m}(T(z)) . \\
P_{m+1}(z)=(z-\lambda) P_{m}(z)-a_{m}^{2} P_{m-1}(z), \quad m=0,1,2, \ldots,
\end{gathered}
$$

where

$$
\begin{aligned}
& \lambda-a_{2 m+1}^{2}-a_{2 m}^{2}=0 \quad m=0,1,2, \ldots \\
& a_{m}^{2}=a_{2 m}^{2} a_{2 m-1}^{2}
\end{aligned}
$$

From these we can derive [6]

$$
\lambda>a_{2^{n}-1}^{2} \geq \lambda-1, \quad n=0,1,2, \ldots .
$$

From (7) and (8) we obtain

$$
\frac{a_{2^{n}}^{2}}{a_{2^{n-1}}^{2}}=\frac{1}{a_{2^{n}-1}^{2}} \leq \frac{1}{\lambda-1}
$$

Hence, since $a_{1}=1$,

$$
a_{2^{n}}^{2} \leq \frac{1}{(\lambda-1)^{n}}
$$

In (5) if we take inner products with $P_{m-1}$ and use orthogonality we obtain

$$
\left\|P_{m}\right\|^{2}=a_{m}^{2}\left\|P_{m-1}\right\|^{2},
$$

so we may prove (1) using the invariance of $T$, (4), and (9).

$$
\left\|P_{2^{n}-1}\right\|^{2}=\frac{\left\|P_{2^{n}}\right\|^{2}}{a_{2^{n}}^{2}}=\frac{\left\|P_{1}\right\|^{2}}{a_{2^{n}}^{2}}=\frac{\lambda}{a_{2^{n}}^{2}} \geq \lambda(\lambda-1)^{n} .
$$


We need only prove the second inequality in (2). We do so by induction. For $n=0$ we have from (4) and (3),

$$
\left\|P_{2}\right\|_{\infty, B}=\left\|P_{1}\right\|_{\infty, B}=\|z-\lambda\|_{\infty, B}=c .
$$

Now suppose the theorem is true for $n=k-1$. If $s$ is even the theorem is true for $2^{k}+s$ using the reduction in (4). If $s$ is odd, use (5) with $m=2^{k}+s$, let $2 j+1=m$, and use (4), the induction hypothesis, and (8) to obtain the last result required,

$$
\begin{aligned}
\left\|P_{2 j+1}\right\|_{\infty, B} & =\left\|\frac{1}{z-\lambda}\left(P_{j+1}(T(z))+a_{2 j+1}^{2} P_{j}(T(z))\right)\right\|_{\infty, B} \\
& \leq \frac{1}{c}\left(\left\|P_{j+1}\right\|_{\infty, B}+\lambda\left\|P_{j}\right\|_{\infty, B}\right) \\
& \leq \frac{1+\lambda}{c}\left(\frac{1+\lambda}{c}\right)^{n-1} .
\end{aligned}
$$

Remark. The combination of the bounds in the theorem tells us that

$$
\lim _{\lambda \rightarrow \infty} \frac{\left\|P_{2^{n}-1}\right\|^{2}}{\lambda^{n+1}}=1 .
$$

M. F. Barnsley was supported by N.S.F. Grant MCS-8104862; J. S. Geronimo and A. N. Harrington were supported by N.S.F. Grant MCS-8203325.

\section{REFERENCES}

[1] G. A. Baker \& P. R. Graves-Morris. Padé Approximants, (Two volumes). Encyclopedia of Mathematics and Its Applications. Addison-Wesley: 1981.

[2] M. F. Barnsley \& A. N. Harrington. Moments of balanced measures on Julia sets. To appear in Trans. Amer. Math. Soc. (1983).

[3] M. F. Barnsley, D. Bessis \& P. Moussa. The Diophantine moment problem and the analytic structure in the activity of the ferromagnetic Ising model. J. Math. Phys. 20 (1979), 535-546.

[4] M. F. Barnsley, J. S. Geronimo \& A. N. Harrington. On the invariant sets of a family of quadratic maps. (Original Aug. 1981.) Shortened version: Comm. Math. Phys. 88 (1983), 479-501.

[5] M. F. Barnsley, J. S. Geronimo \& A. N. Harrington. Orthogonal polynomials associated with invariant measures on Julia sets. Bull. Amer. Math. Soc. 7 (1982), 381-384.

[6] M. F. Barnsley, J. S. Geronimo \& A. N. Harrington. Some treelike Julia sets and Padé approximants. Letters in Math. Phys. 7 (1983), 279-286.

[7] M. F. Barnsley, J. S. Geronimo \& A. N. Harrington. Infinite dimensional Jacobi matrices associated with Julia sets. Proc. Amer. Math. Soc. 88 (1983), 625-630.

[8] M. F. Barnsley, J. S. Geronimo \& A. N. Harrington. Geometrical and electrical properties of some Julia sets. To appear in an anthology edited by G. V. and D. Chudnovsky.

[9] J. Bellissard, D. Bessis \& P. Moussa. Chaotic states for almost periodic Shrödinger operators. Phys. Rev. Letters 49.(1982), 701-704.

[10] D. Bessis \& P. Moussa. Orthogonality properties of iterated polynomial mappings. Comm. Math. Phys. 88 (1983), 503-529.

[11] D. Bessis, M. L. Mehta \& P. Moussa. Orthogonal polynomials on a family of Cantor sets and the problem of iterations of quadratic mappings. Letters in Math. Phys. 6 (1982), 123-140.

[12] H. Brolin. Invariant sets under iteration of rational functions. Arkiv för Matematik 6, (1965), 103-144.

[13] P. Collet \& J. Eckmann, Iterated Maps on the Interval as Dynamical Systems. Birkhauser: BaselBoston, 1980.

[14] S. Demko. In preparation. 
[15] M. P. Fatou. Sur les equations fonctionnelles. Bull. Soc. Math. de France 47 (1919), 161-271; ibid. 48, 33-94; ibid. 48, 208-314.

[16] C. Grebogi, E. Ott \& J. A. Yorke. Chaotic attractors in crisis. Phys. Rev. Letters 48 (1982), 1507-1510.

[17] M. V. Jakobson. Absolutely continuous invariant measures for one parameter families of onedimensional maps. Comm. Math. Phys. 81 (1981), 39-88.

[18] R.-J. de Jonkere. Convergence de l'iteration des fonctions rationelles. Thesis, (1963), Université Catholique de Louvain, Faculté des Sciences Appliqueés.

[19] G. Julia. Mémoire sur l'iteration des fonctions rationelles. Journal de Math. Pures et Appliqueés 4 (1918), 47-245.

[20] W. Rudin. Real and Complex Analysis. McGraw-Hill: New York, 1966.

[21] D. Sullivan. Iteration des fonctions analytiques complexes. C.R. Acad. Sci. Paris 294 (1982), 301-303. 